\title{
Snowboarding injuries: a review of the literature and an analysis of the potential use of portable ultrasound for mountainside diagnostics
}

\author{
M. R. Nowak • A. W. Kirkpatrick • \\ J. A. Bouffard · D. Amponsah · S. A. Dulchavsky
}

Published online: 7 January 2009

(c) The Author(s) 2008. This article is published with open access at Springerlink.com

\begin{abstract}
Snowboarding has become a popular recreational and professional sport. Participants suffer a variety of injuries, especially of the extremities, that require medical evaluation. This article reviews the reported injuries to both leisure and elite athletes. To many, an injured extremity requires travel to a medical facility for accurate evaluation. Musculoskeletal ultrasound is an accurate and portable technology that can be used for real time, mountainside diagnoses of these injuries.
\end{abstract}

Keywords Mountainside ultrasound - Remote guidance ultrasound - Snowboarding injuries · Diagnostic ultrasound · Mountainside diagnostics

M. R. Nowak ( $\square)$

Departments of Emergency Medicine and Surgery,

Henry Ford Hospital, Detroit, MI, USA

e-mail: mnowak2@hfhs.org

A. W. Kirkpatrick

Regional Trauma Services, Foothills Medical Center,

Calgary, AB, Canada

J. A. Bouffard

Department of Radiology, Henry Ford Hospital, Detroit, MI, USA

D. Amponsah

Department of Emergency Medicine, Henry Ford Hospital,

Detroit, MI, USA

S. A. Dulchavsky

Department of Surgery, Henry Ford Hospital, Detroit, MI, USA

\section{Introduction}

Snowboarding has experienced a tremendous growth as a sport over the years. The National Sporting Goods Association reported 6.6 million snowboarders participating in this sport in 2004. To-date there has not been a careful overall review of the medical literature to summarize the injuries sustained by these individuals. Snowboarding is physically demanding and is often conducted in a challenging physical environment such that the risks of serious injury or even death are real. Reviews of snowboarding injuries from around the world have demonstrated head, abdominal, spinal, and extremity trauma to be the most frequent serious, and occasionally fatal, injuries sustained [1-4]. Fortunately, most snow-boarding injuries are not life-threatening, but when a professional or leisure snowboarder suffers a potential traumatic injury, it can be extremely challenging for a non-physician on-mountain care-provider to make the correct diagnosis. This is because of the limited medical knowledge of the mountain patrol or sports trainers and the lack of radiological imaging support. Often the snowboarder must be transported to a local hospital or to a specialty clinic for additional medical assessments [5].

Generally, conditions in which snowboarders are injured preclude the ability to quickly assess injuries using imaging techniques such as magnetic resonance imaging (MRI), CAT scans, and even plain radiographs. As many snowboarding facilities are located in relatively remote locations, an initial accurate diagnostic assessment would be critical to maximize the recovery of a patient. For example, in the case of an individual who suffers a potential joint injury of unknown severity, the decision must be made to apply first aid principles (the application of cold and elevation to the affected area, anti-inflammatory medications, and rest for a 
day or so) or to send the individual to a hospital for further diagnostic evaluation.

There are similarities between injured astronauts and snowboarders in that both may be extremely limited in the available medical and diagnostic resources that can be provided. In order to address this challenge, NASA has refined techniques of remote guidance ultrasound (US) technique to assist onboard non-physician care-providers to help make accurate and quick initial diagnoses aboard the International Space Station (ISS) [5-7]. While astronauts perform US on the ISS, doctors on earth are able to view and guide the US examination in real time. When provided with two-way voice and single-direction video communications, remote experts can immediately interpret the images and provide feed-back and remote guidance to the novice on-board users to obtain the best possible images and thus answer a clinical question at hand. These physicians can then decide whether an injured astronaut can be medically managed in space or needs to be brought back to earth for hospital care [8]. On mountain slopes this technology could also be utilized to advance the medical care of injured snowboarders. If remotely supported using such tele-ultrasound techniques, we propose that trained snow patrol teams or individual trainers could use a portable US machine to immediately diagnose injuries that previously required the delayed use of large radiographic machines for evaluation. Whether a hospital transfer was necessary might be decided depending on the mountain patrol or the trainer's US diagnosis. If obvious pathology was detected then evacuation should be expedited. If a less experienced operator was still suspicious of a negative examination or if an operator wished to confirm their diagnosis, they could upload the US images and send them to the appropriate radiologic experts anywhere on the globe for consultation. This article thus reviews the traumatic snowboard injuries that have been reported to-date and discusses the potential use of US technology as a diagnostic tool for mountainside determination of the extent of snowboarding injuries.

\section{Snowboarding injuries in elite and recreational riders}

A study of elite Norwegian snowboarders showed that overall injury risk is high and that the traumas suffered by these athletes are different from those seen among recreational snowboarders. Specifically the elite group reported fewer wrist injuries but more knee and back traumas. A follow-up study was performed to confirm these findings at the last race in March 2003 at the Federation Internationale de Ski Snowboard World Cup held in Switzerland. Acute injuries resulting in missed participation and traumas
Federation Internationale de Ski Snowboard World Cup Injuries Snowboard Injury Broakdown

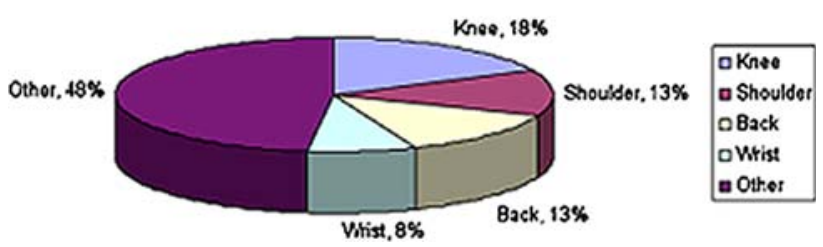

Fig. 1 Injury breakdown at the Federation Internationale de Ski Snowboard World Cup

influencing performance were recorded during an interview. Exposure was reported as the number of runs in all disciplines and the incidence rate was calculated as number of injuries per 1,000 runs [9]. At the Federation Internationale de Ski Snowboard World Cup, 258 elite snowboarders were interviewed after participating in a total number of 3,193 competition days in all disciplines. They reported suffering 158 injuries (some with more than one) with only 62 of these traumas occurring in the actual competitions. The most common injuries were located in the knee $(n=24 ; 18 \%)$, shoulder $(n=18 ; 13 \%)$, back $(n=17 ; 13 \%)$, and wrist $(n=11 ; 8 \%)$ (Fig. 1).

The total competition exposure was estimated to be 46,879 runs based on the fixed exposure factors estimated for each discipline. Therefore the calculated injury incidence rate was 1.3 traumas per 1,000 runs.

One of the main difficulties in obtaining accurate data about recreational sports injuries is that they occur in a mobile population. Many of these participants often wait until they return home before having their injuries evaluated [10]. The overall injury rate amongst recreational snowboarders is estimated to be 4-6 injuries per 1,000 snowboarder days, which is slightly higher than that seen in non-elite skiers. Over half $(53 \%)$ of all injuries in the recreational snowboarder occur in the upper extremity [11]. Additionally, $15 \%$ of traumatic cases are head injuries, composed mostly of concussions. A review of 3,645 upper extremity injuries in snowboarders detailed the specific emerging trends in their traumas. A majority $(21.6 \%)$ of these injuries occurred in the wrist, $16.2 \%$ in the shoulder, $4.1 \%$ in the hand, $3.8 \%$ in the elbow, and $2.7 \%$ in the forearm region. These upper extremity injuries are more common in beginners as advanced snowboarders are more likely to have complex and severe wrist fractures from extremely severe falls. Lower extremity trauma, although not as common as upper extremity injury, is also frequently seen. Of these the ankle accounts for $12-28 \%$, the knee for $13-23 \%$, and the foot for $1-3 \%$ [12]. 
Specific upper extremity traumatic snowboarding injuries that have been reported are as follows. Distal radius fractures occur in those who are less experienced and are not usually wearing wrist guards. This injury typically occurs when the outstretched hand is used in an attempt to break a fall. Scapholunate dissociation presents as pain in the dorsal aspect of the wrist, also after falling onto an outstretched hand. Shoulder dislocation, which accounts for $8-16 \%$ of snowboarding injuries, commonly occurs from falling forward (toward the toe edge) on a snowboard. Other less common injuries to the shoulder include rotator cuff strains or tears, glenohumeral subluxations and dislocations, acromioclavicular separations, and clavicular fractures. Elbow injuries account for 2-5\% of injuries and include contusions, sprains, strains, fractures, and dislocations. These elbow injuries likewise usually result from a drop onto an outstretched arm. Forearm injuries, although uncommon, can be seen after a severe fall. These include Monteggia fractures/dislocations (fracture of the proximal ulna with associated dislocation of the proximal radius) and Galeazzi fracture/ dislocations (fracture of the radius at the junction of the mid and distal one-third associated with dislocation of the distal radioulnar joint) [12].

Specific lower extremity snowboarding trauma that has been reported is as follows. A fracture of the lateral process of the talus (LPT) has been known as the "snowboarder's fracture" [13] and is reported to represent $15 \%$ of all ankle snowboarding injuries. This previously rare injury has become more common, and typically occurs when a snowboarder lands hard from a jump with his body weight too far over the toe edge of the board. Ankle ligamentous sprains are commonly seen (most are of the lateral ankle ligaments) and half of all the injuries to the ankle are sprains. Knee injuries, although more common in skiers, include cruciate and collateral ligament injuries from falling at high speed, collisions, and while chair lift uploading and unloading. Anterior cruciate ligament (ACL) sprains occur while medial collateral ligament (MCL) injuries are not often seen in snowboarding given the positioning of the feet on the board [12].

There are a number of non-extremity traumas that have also been associated with snowboarding. Spinal injuries account for significant injuries (0.004/1,000 snowboarding days) and are commonly related to falls or errors while jumping and landing [4]. Chest injuries account for $1-6 \%$ of all snowboarding injuries and include rib fractures $(55.2 \%)$, rib contusions $(37.5 \%)$, sternal fractures $(3.1 \%)$, pneumothorax/hemothorax $(3.1 \%)$, and pneumomediastinum (1.1\%). Abdominal injuries account for $2-7 \%$ of injuries and occur from falling (53\%), errors occurring during jumping (32\%), and collisions (16\%) [12].

\section{The potential of ultrasound use in the diagnosis of snowboarding injuries}

We believe the vast majority of all reported snowboarding injuries could be assessed and accurately diagnosed mountainside utilizing portable US imaging. The use of US to supplement the physical examination of the injured patient is becoming increasingly accepted as an additional basic "sense" to complement inspection, auscultation, and palpation. An US enhanced initial assessment can address serious life-threatening pathology of the airway, respiratory, and circulatory systems, and completely complements the primary survey of the Advanced Trauma Life Support Course [14]. However, given the medical complexity of abdominal and thoracic trauma, and the catastrophic consequences a misdiagnosis of visceral injury or organ system instability, we believe the initial mountainside use of US in the hands of non-physicians should be limited to the evaluation of extremity trauma. Such injuries have been studied extensively using musculoskeletal US techniques, accurately showing the extent and specific location of the injury [4]. While it is impossible to document the specifics of each sonographic evaluation for all the extremity snowboard injuries reported, three examples follow which show its potential use close to the scene of the accident.

\section{Case reports}

\section{Case 1}

The first case shows the US probe placement and the resultant sonographic image seen in a patient suffering a fracture of the left second metacarpal bone (Fig. 2).

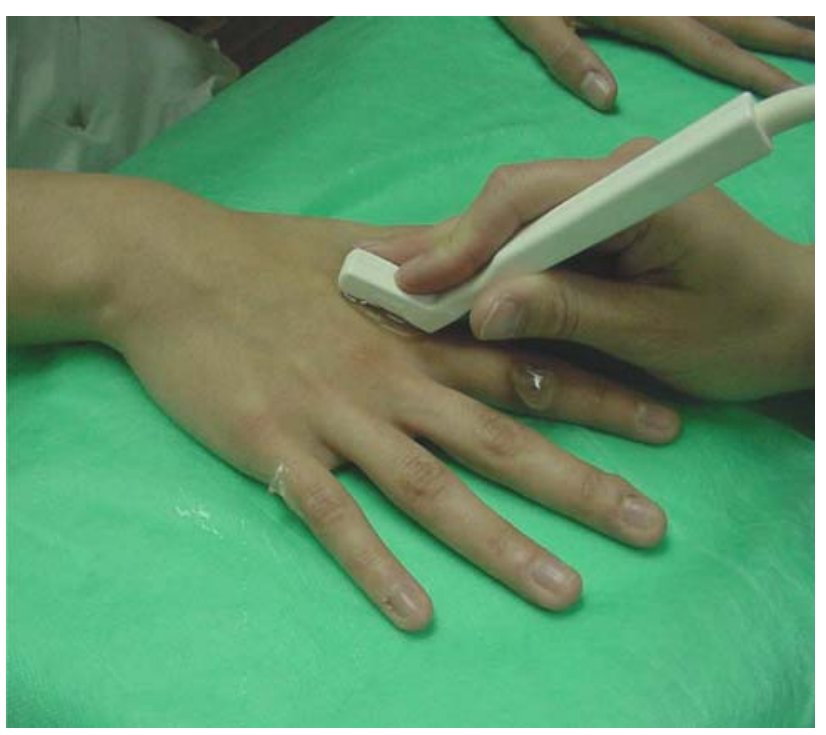

Fig. 2 US probe placement and the resultant sonographic image seen in a patient suffering a fracture of the left second metacarpal bone 


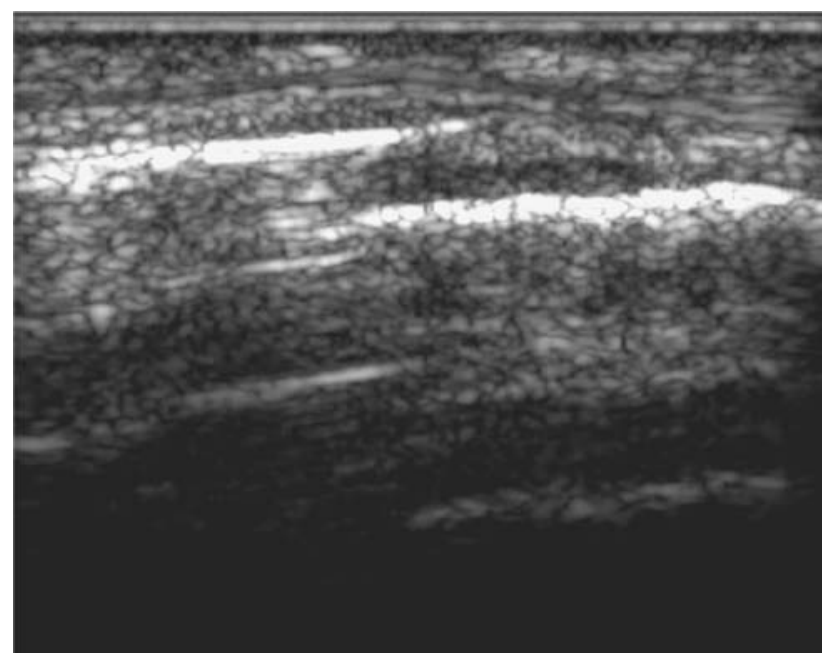

Fig. 3 Long-axis scan of a hand injury showing a fracture of the left second metacarpal bone

The fracture is visualized as a disruption of the hyperechoic cortical surface, specifically as a separation or stepoff deformity along the cortical surface (Fig. 3).

\section{Case 2}

The second case shows the mechanism of injury, US probe placement, and the resultant sonographic image seen in a snowboarder suffering an acute subscapularis tendon tear, a rotator cuff injury (Figs. 4, 5, 6).

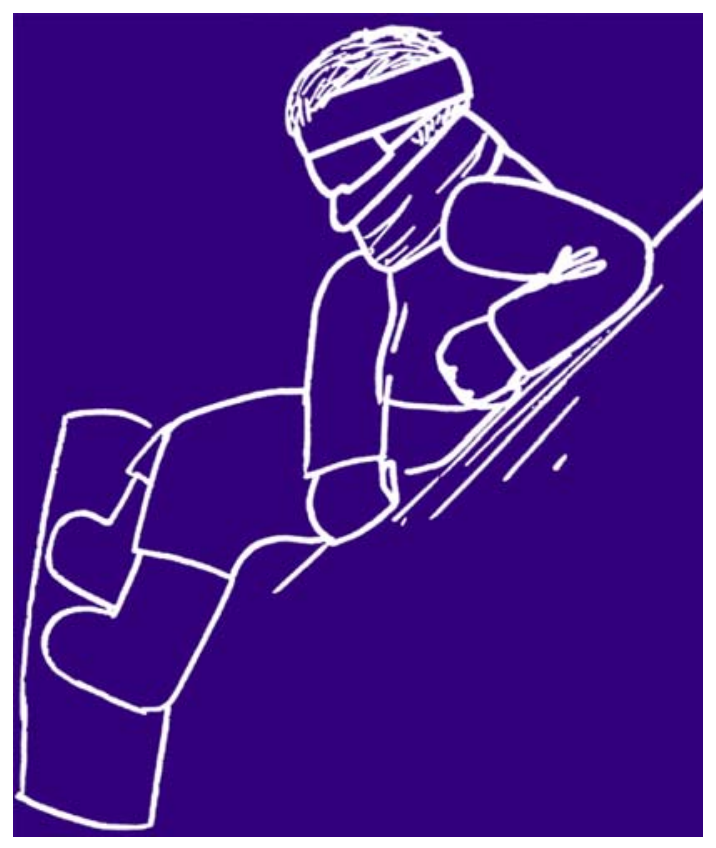

Fig. 4 Mechanism of shoulder injury in a snowboarder falling backwards

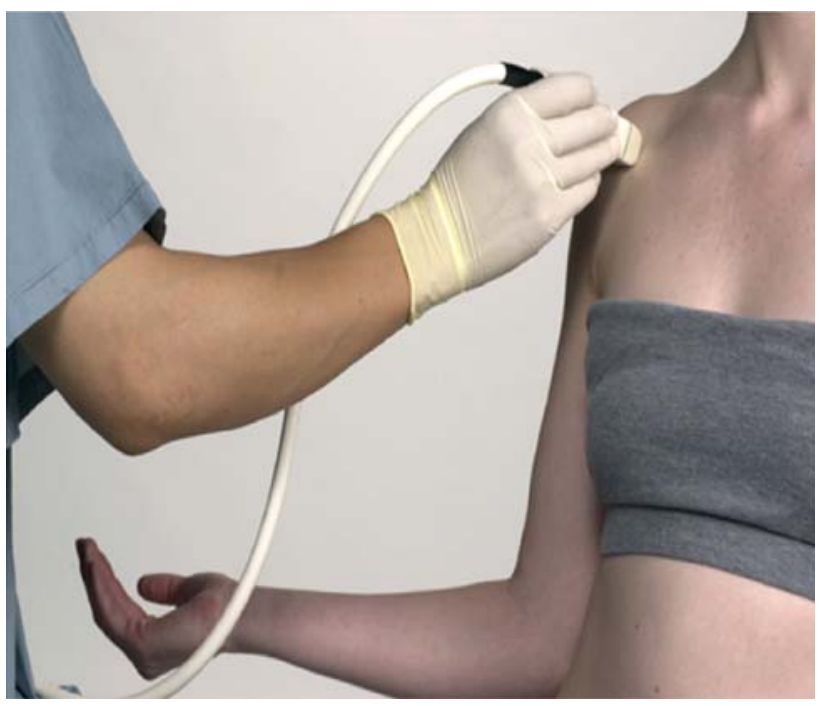

Fig. 5 Short-axis sonographic view showing the patient with the shoulder being evaluated in an externally rotated position

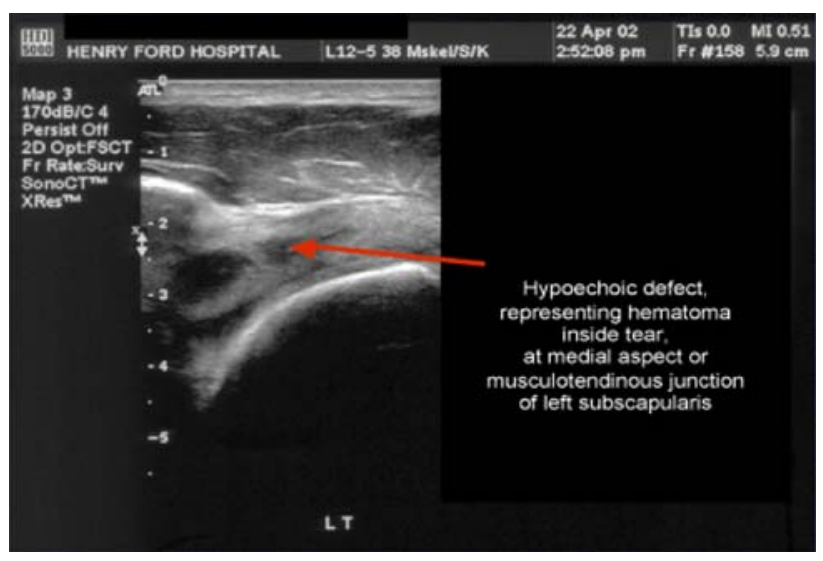

Fig. 6 Short axis sonographic shoulder view showing an acute subscapular tendon tear

\section{Case 3}

This case shows one of the authors (Kirkpatrick AW) using portable ultrasound mountainside to evaluate the injury suffered to the knee of a snowboarder. This demonstrates the feasibility of actually doing real time ultrasound evaluation at or near the site of injury (Fig. 7).

\section{Conclusions}

Snowboarding, whether at the elite or recreational level, is associated with significant injuries involving the abdomen, chest, head, and extremities. Portable mountainside US has the potential to accurately identify the extremity injuries 


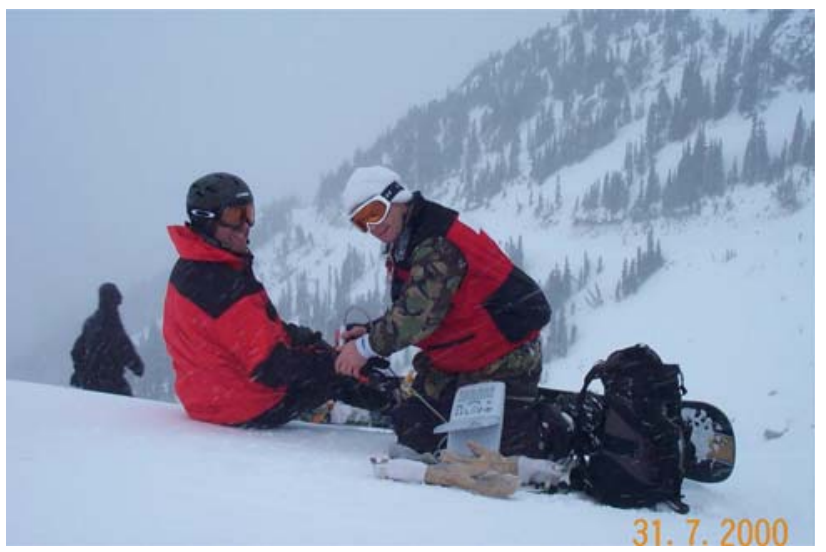

Fig. 7 This is an illustrative example of the portability of ultrasound allowing it to be used as a real-time tool to evaluate injured snowboarders on the mountain

involved. This is very important at the elite level as decisions can be made as to whether the trauma requires only some local care with early return to training or competition, or whether the athlete requires transfer to a hospital facility for further imaging and medical evaluation. Preliminary studies involving the USA Women's Olympic Hockey Team and some of the professional sports teams in Detroit (Red Wings Hockey, Lions Football) prove this concept is both feasible and valuable for the evaluations of injuries suffered by these athletes (personal communication, Dr. Scott A. Dulchavsky). Further, the use of portable US for the evaluation of recreational snowboarding injuries or other sports related trauma needs further evaluation. Making the correct diagnosis mountainside in these more recreational cases could eliminate a misdiagnosis resulting in further injury and suffering, or a trip to a more distant facility for unnecessary negative imaging studies. While more studies are required before the widespread application of portable US use in the evaluation of snowboarding and other sports injuries, its potential use in these circumstances remains very attractive.
Open Access This article is distributed under the terms of the Creative Commons Attribution Noncommercial License which permits any noncommercial use, distribution, and reproduction in any medium, provided the original author(s) and source are credited.

\section{References}

1. Corra S, et al. Skiing and snowboarding injuries and their impact on the emergency care system in South Tyrol: a restrospective analysis for the winter season 2001-2002. Inj Control Saf Promot. 2004;11(4):281-5.

2. Federiuk CS, Schlueter JL, Adams AL. Skiing, snowboarding, and sledding injuries in a northwestern state. Wilderness Environ Med. 2002;13(4):245-9.

3. Prall JA, Winston KR, Brennan R. Severe snowboarding injuries. Injury. 1995;26(8):539-42.

4. Tarazi F, Dvorak MF, Wing PC. Spinal injuries in skiers and snowboarders. Am J Sports Med. 1999;27(2):177-80.

5. Kwon D, et al. Battling fire and ice: remote guidance ultrasound to diagnose injury on the International Space Station and the ice rink. Am J Surg. 2007;193(3):417-20.

6. Fincke EM, et al. Evaluation of shoulder integrity in space: first report of musculoskeletal US on the International Space Station. Radiology. 2005;234(2):319-22.

7. Foale CM, et al. Diagnostic instrumentation aboard ISS: just-intime training for non-physician crew members. Aviat Space Environ Med. 2005;76(6):594-8.

8. Sargsyan A, et al. FAST at MACH 20: clinical ultrasound aboard the International Space Station. J Trauma. 2005;58(1):35-9.

9. Torjussen J, Bahr R. Injuries among elite snowboarders (FIS Snowboard World Cup). Br J Sports Med. 2006;40(3):230-4.

10. Crim J. Winter sports injuries. The 2002 Winter Olympics experience and a review of the literature. Magn Reson Imaging Clin N Am. 2003;11(2):311-21.

11. Idzikowski JR, Janes PC, Abbott PJ. Upper extremity snowboarding injuries ten-year results from the Colorado snowboard injury survey. Am J Sports Med. 2000;28(6):825-32.

12. Moeller J, Rifat SF. Winter sports medicine handbook. New York: McGraw-Hill; 2004.

13. Kirkpatrick DP, et al. The snowboarder's foot and ankle. Am J Sports Med. 1998;26(2):271-7.

14. Kirkpatrick AW. Clinician-performed focused sonography for the resuscitation of trauma. Crit Care Med. 2007;35(5 Suppl): S162-72. 